\title{
One-loop corrections to multiscale effective vertices in the EFT for Multi-Regge processes in QCD
}

\author{
Maxim Nefedov*† \\ Samara National Research University, \\ II Institute for Theoretical Physics, Hamburg University \\ E-mail: nefedovma@gmail.com
}

\begin{abstract}
The computation of one-loop corrections to the $\gamma^{\star} Q_{+} q$ and $g R_{+} g$ effective vertices in the framework of gauge-invariant effective theory for Multi-Regge processes in QCD is reviewed. Due to consistent implementation of the "tilted Wilson line" regularization for rapidity divergences, the gauge-invariance has been preserved at all stages of calculation independently on the rapidity regulator and cancellation of the power-like dependence on the regularization variable is traced. Only single-logarithmic rapidity divergence is left in the final result.
\end{abstract}

XXVII International Workshop on Deep Inelastic Scattering and Related Subjects Torino, Italy, 8-12 April 2019

\footnotetext{
* Speaker.

$\dagger$ Work supported in part by the Foundation for the Advancement of Theoretical Physics and Mathematics BASIS, grant No. 18-1-1-30-1
} 


\section{Introduction}

In the Multi-Regge Kinematics (MRK) for the $2 \rightarrow 2+n$ partonic scattering in QCD, the final-state partons can be grouped into clusters w.r.t. their rapidity. Different clusters are highlyseparated in rapidity from each-other, so that the typical $t$-channel momentum transfer is much smaller than the invariant mass of any pair of final-state clusters. At leading power in $t / s$, higherorder QCD corrections to such amplitudes are enhanced by high-energy logarithms $\log s /(-t)$. The Gauge-Invariant Effective Field Theory (EFT) for Multi-Regge processes in QCD [1, 2] has been introduced as a systematic tool for computation of asymptotics of QCD scattering amplitudes in the Multi-Regge limit in the Leading Logarithmic Approximation and beyond. The Hermitian version of this EFT [3,4] contains all corrections, restoring the unitarity of High-Energy scattering and therefore provides a framework for studies of High-Energy QCD and gluon saturation phenomena, alternative to the Balitsky-JIMWLK or Color-Glass Condensate pictures, see Refs. [5, 6] for the recent work in this direction.

In the High-Energy EFT [1, 2], different rapidity-clusters of final-state particles are produced by different gauge-invariant subamplitudes - effective vertices. This effective vertices are connected by $t$-channel exchanges of Reggeized gluons $\left(R_{ \pm}\right)$and Reggeized quarks $\left(Q_{ \pm}\right)$, collectively named as Reggeons - gauge-invariant degrees of freedom of the High-Energy QCD. Eventually, it should be possible to integrate-out physical quarks and gluons, order-by-order in $\alpha_{s}$, and formulate the high-energy limit of QCD entirely in terms of Reggeons - Reggeon Field Theory, see e.g. $[6,7,8]$. Calculation of the one-loop corrections to different effective vertices is a major task in development of this formalism.

The main technical difficulty in the Higher-Order calculations in High-Energy EFT is the appearance of Rapidity divergences in loop and phase-space integrals. These divergences arise due to the presence of "Eikonal" denominators $1 / l^{ \pm}$in the induced vertices of interactions of Reggeons with ordinary (Yang-Mills) partons, taken together with kinematical constraints following from MRK. See Sec. 2 of Ref. [9] for the analysis of the conditions of appearance of rapidity divergences at one loop. At present, many calculations $[9,10,11,12]$ in the High-Energy EFT has been done with the use of a variant of "tilted Wilson line" regularization, where the direction vectors $\left(n_{\mu}^{ \pm}\right)$ of Wilson lines in the definition of Reggeon-parton interactions are slightly shifted from the lightcone:

$$
n_{\mu}^{ \pm} \rightarrow \tilde{n}_{\mu}^{ \pm}=n_{\mu}^{ \pm}+r \cdot n_{\mu}^{\mp}, \frac{1}{l_{ \pm}} \rightarrow \frac{1}{\tilde{l}_{ \pm}}=\frac{1}{l_{ \pm}+r \cdot l_{\mp}},
$$

where $0<r \ll 1$ is the regularization variable. In Ref. [9] we have observed, that to keep the $R g$ interaction gauge-invariant for $r \neq 0$ one also have to modify the usual MRK kinematic constraint, stating that four-momentum $q_{1}$ of $R_{+}$-Reggeon has only one nonzero light-cone component $q_{1}^{+}$ and transverse momentum $\mathbf{q}_{T 1}$. The kinematic constraint for Reggeon $R_{+}$, consistent with gaugeinvariance at $r \neq 0$ is

$$
\tilde{q}_{1}^{-}=q_{1}^{-}+r \cdot q_{1}^{+}=0
$$

For Reggeized quarks, such modification is not strictly necessary, but it turns out, that many scalar integrals actually simplify in the kinematics (1.2), so we prefer to keep it both for Reggeized gluons and quarks. 
In the present contribution we will discuss two examples of one-loop corrections to ReggeonParticle-Particle effective vertices: $\gamma^{\star} Q_{+} q$ and $g R_{+} g$. The first one involves an off-shell photon $\left(\gamma^{\star}\right)$, so that the vertex has two scales of virtuality: virtuality of the photon $q^{2}=-Q^{2}<0$ and of the Reggeized quark $q_{1}^{2}=-t_{1}<0$. More details concerning this example can be found in our Ref. [9]. The second example already has been considered in Ref. [10], however in this reference part of diagrams has been but to zero by the gauge choice for external gluons and therefore gaugeinvariance of amplitude and cancellation of power-like dependence on the rapidity-regulator $r$ has not been verified. We fill this gap in the present contribution.

Our paper has the following structure: In the Sec. 2 integrals appearing in our calculation are listed and we comment on their rapidity divergences. Explicit expressions for this integrals are provided in Ref. [9]. In Sec. 3 we review the calculations for above-mentioned examples and in the Sec. 4 we summarize our conclusions.

\section{One-loop rapidity-divergent integrals}

It is convenient to categorize one-loop integrals appearing in our calculations according to the type of their dependence on the rapidity-regulator variable $r$. Then the simplest integrals containin only one quadratic and one or two linear propagators:

$$
A_{[-]}(p)=\int \frac{\left[d^{d} l\right]}{(p+l)^{2}\left[\tilde{l}^{-}\right]}, A_{[--]}(p)=\int \frac{\left[d^{d} l\right]}{l^{2}\left[\tilde{l}^{-}\right]\left[\tilde{l}^{-}-\tilde{p}^{-}\right]},
$$

are related with each-other as:

$$
A_{[--]}(p)=\frac{1}{\tilde{p}_{-}} A_{[-]}(p),
$$

where $\left[d^{d} l\right]=\left(\mu^{2}\right)^{\varepsilon} d^{d} l /\left(i \pi^{d / 2} r_{\Gamma}\right), d=4-2 \varepsilon, r_{\Gamma}=\Gamma^{2}(1-\varepsilon) \Gamma(1+\varepsilon) / \Gamma(1-2 \varepsilon)$ and $1 /[X]$ denotes the PV-prescription, and both integrals are proportional to $r^{-1+\varepsilon}$.

Integrals

$$
B_{[-]}(p)=\int \frac{\left[d^{d} l\right]}{l^{2}(p+l)^{2}\left[\tilde{l}^{-}\right]}, \quad B_{[--]}(p)=\int \frac{\left[d^{d} l\right]}{l^{2}(p+l)^{2}\left[\tilde{l}^{-}\right]\left[\tilde{l}^{-}+\tilde{p}^{-}\right]},
$$

are related as:

$$
B_{[--]}(p)=\frac{2}{\tilde{p}_{-}} B_{[-]}(p),
$$

and contain the $r^{\varepsilon}$-dependence on the rapidity regulator for $p^{2}=0$ and also term $\propto r^{-\varepsilon}$ appears for $p^{2} \neq 0$. Cancellation of power-like terms happens between different diagrams and hence is a nontrivial dynamical property of QCD.

The integral

$$
B_{[+-]}(p)=\int \frac{\left[d^{d} l\right]}{l^{2}(p+l)^{2}\left[\tilde{l}^{+}\right]\left[\tilde{l}^{-}\right]},
$$

contributes to one-loop correction to propagators of Reggeized gluon and quark and it contains only logarithmic rapidity-divergence $\sim \log r$, related with Reggeization. Similar single-logarithmic divergence is present in a "triangle" integral:

$$
C_{[-]}\left(-q_{1}^{2},-q^{2}, q^{-}\right)=\int \frac{\left[d^{d} l\right]}{l^{2}\left(q_{1}+l\right)^{2}\left(q_{1}+q+l\right)^{2}\left[\tilde{l}^{-}\right]},
$$




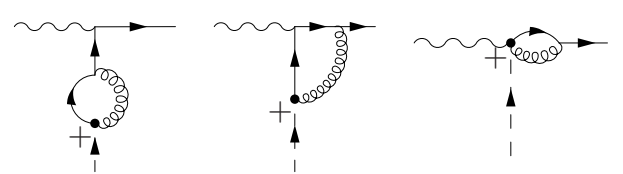

Figure 1: Diagrams contributing to the $\gamma^{\star} Q q$-vertex at one loop. Dashed line with an arrow - Reggeized quark.

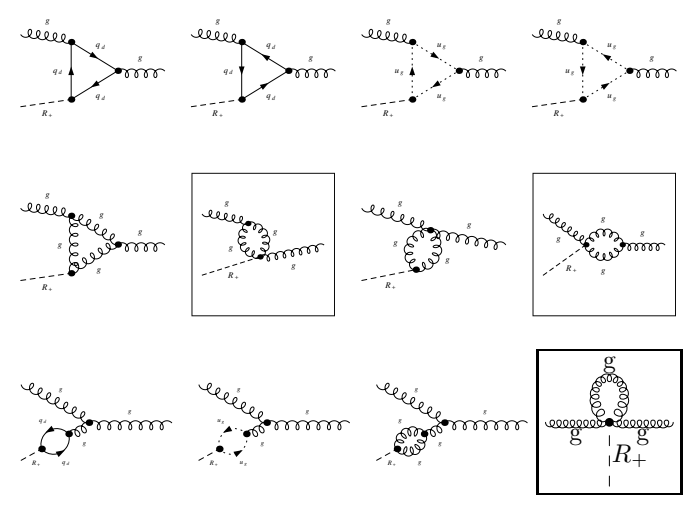

Figure 2: Diagrams contributing to the $g R_{+} g$-vertex at one loop. Dashed line - Reggeized gluon, dotted line - Faddeev-Popov ghost.

which has been computed for the case $q^{2}=0$ in Ref. [10] and for the case $q^{2} \neq 0$ in the Ref. [9]. For the $q^{2} \neq 0$ case, the term $\propto r^{-\varepsilon}$ also appears in the integral $C_{[-]}$.

\section{One-loop effective vertices}

The set of EFT Feynman diagrams, contributing to the one-loop correction to $\gamma^{\star} Q_{+} q$-effective vertex is shown in the Fig. 1. The result [9]:

$$
\Gamma_{+\mu}^{(1)}\left(q_{1}, q\right)=i e e_{q} \cdot \bar{u}\left(q+q_{1}\right)\left[C[\Gamma] \cdot \Gamma_{+\mu}^{(0)}\left(q_{1}, q\right)+C\left[\Delta^{(1)}\right] \cdot \Delta_{+\mu}^{(1)}\left(q_{1}, q\right)+C\left[\Delta^{(2)}\right] \cdot \Delta_{+\mu}^{(2)}\left(q_{1}, q\right)\right],
$$

can be expressed in terms of three gauge-invariant Lorentz structures:

$$
\Gamma_{+\mu}^{(0)}\left(q_{1}, q\right)=\gamma_{\mu}+\frac{\hat{q}_{1} n_{\mu}^{-}}{2 q^{-}}, \Delta_{+\mu}^{(1)}\left(q_{1}, q\right)=\frac{\hat{q}}{q_{-}}\left(n_{\mu}^{-}-\frac{2\left(q_{1}\right)_{\mu}}{q_{1}^{+}}\right), \Delta_{+\mu}^{(2)}\left(q_{1}, q\right)=\frac{\hat{q}}{q_{-}}\left(n_{\mu}^{-}-\frac{q_{\mu}}{q^{+}}\right),
$$

where $\Gamma_{+\mu}^{(0)}$ is the Fadin-Sherman scattering vertex and coefficients are the following:

$$
\begin{aligned}
C[\Gamma]= & -\frac{\bar{\alpha}_{s} C_{F}}{4 \pi} \frac{1}{2}\left\{\frac{\left[(d-8) Q^{2}+(d-6) t_{1}\right] B\left(t_{1}\right)-2(d-7) Q^{2} B\left(Q^{2}\right)}{Q^{2}-t_{1}}\right. \\
& \left.-2\left[\left(Q^{2}-t_{1}\right) C\left(t_{1}, Q^{2}\right)-q_{-}\left(t_{1} C_{[-]}\left(t_{1}, Q^{2}, q_{-}\right)+\left(B_{[-]}(q)-B_{[-]}\left(q+q_{1}\right)\right)\right)\right]\right\}, \\
C\left[\Delta^{(1)}\right]= & -\frac{\bar{\alpha}_{s} C_{F}}{4 \pi} \frac{\left(Q^{2}+t_{1}\right)}{2\left(Q^{2}-t_{1}\right)^{2}}\left[\left((d-2) Q^{2}-(d-4) t_{1}\right) B\left(t_{1}\right)-2 Q^{2} B\left(Q^{2}\right)\right], \\
C\left[\Delta^{(2)}\right]= & -\frac{\bar{\alpha}_{S} C_{F}}{4 \pi} \frac{Q^{2}}{\left(Q^{2}-t_{1}\right)^{2}}\left[\left((d-6) t_{1}-(d-8) Q^{2}\right) B\left(Q^{2}\right)+2\left(t_{1}-2 Q^{2}\right) B\left(t_{1}\right)\right],
\end{aligned}
$$


were $\bar{\alpha}_{s}=\mu^{-2 \varepsilon} g_{s}^{2} r_{\Gamma} /(4 \pi)^{1-\varepsilon}$ is the dimensionless strong-coupling constant, $B\left(t_{1}\right)$ and $C\left(t_{1}, Q^{2}\right)$ are the usual one-loop scalar "bubble" and "triangle" integrals [13]. We observe, that integrals $A_{[-]}(q)$ appearing in the expansion of the second and third diagrams in the Fig. 1 cancel-away. Also the terms $\propto r^{ \pm \varepsilon}$ cancel between integrals $B_{[-]}(q), B_{[-]}\left(q+q_{1}\right)$ and $C_{[-]}\left(t_{1}, Q^{2}, q_{-}\right)$in Eq. (3.1), so that only single-logarithmic rapidity divergence is left. In Ref. [9] we have checked, that this rapidity divergence cancels in the single-Reggeon exchange contribution to the $\gamma^{\star}+\gamma \rightarrow q+\bar{q}$ amplitude at one loop and EFT result agrees with MRK limit of one-loop QCD amplitude.

Diagrams contributing to the one-loop correction to $g R_{+} g$-vertex with on-shell external YangMills gluons $g$ with helicities $\lambda_{1}$ and $\lambda_{2}$ and momenta $q$ and $q+q_{1}$ are shown in the Fig. 2. This one-loop correction can be decomposed as:

$$
\gamma_{\lambda_{1}+\lambda_{2}}^{a b c,(1)}=i g_{s} f^{a b c} \cdot \varepsilon^{\mu}\left(q, \lambda_{1}\right)\left(\varepsilon^{*}\left(q+q_{1}, \lambda_{2}\right)\right)^{v}\left[C\left[\gamma_{+}^{(0)}\right] \cdot \gamma_{\mu,+, v}^{(0)}+C\left[\delta_{+}\right] \cdot \delta_{\mu,+, v}\right]
$$

where the helicity-conserving (Lipatov's) and helicity-flip Lorentz structures are:

$$
\gamma_{\mu,+, v}^{(0)}=2 q_{-} g_{\mu v}+2 n_{-\mu} q_{1 v}-2 n_{-v} q_{1 \mu}+\frac{t_{1} n_{-\mu} n_{-v}}{q_{-}}, \delta_{\mu,+, v}=2 q_{-}\left[g_{\mu v}+\frac{2 q_{1 \mu} q_{1 v}}{t_{1}}\right],
$$

while coefficients in front of them read:

$$
\begin{aligned}
C\left[\gamma_{+}^{(0)}\right] & =-\frac{\bar{\alpha}_{s} C_{A}}{4 \pi}\left[q_{-} t_{1} C_{[-]}\left(t_{1}, 0, q_{-}\right)+B\left(t_{1}\right)\right], \\
C\left[\delta_{+}\right] & =\frac{\bar{\alpha}_{s}}{4 \pi} \frac{(d-4) B\left(t_{1}\right)}{2(d-1)(d-2)}\left(2 n_{F}-(d-2) C_{A}\right) .
\end{aligned}
$$

Eqns. (3.4) and (3.5) coincide with the results of Ref. [10], however in the calculations in this paper, the diagrams framed in the Fig. 2 where nullified by the gauge-choice for external gluons. We take them into account, and hence we can check the Slavnov-Taylor identities and trace-out the cancellation of power-like dependence on the regulator $r$. Modified kinematical constraint (1.2) guarantees the gauge-invariance of amplitude in all orders in $r$, and we observe, that contributions of integrals $A_{[-]}(q)$ and $B_{[-]}(q)$ cancel in the $O\left(r^{1}\right)$ and $O\left(r^{0}\right)$ respectively, while in higher orders in $r$ (which we eventually drop), coefficients in front of this integrals are gauge-invariant, which serves as a useful cross-check of the calculation. Cancellation of contributions of this integrals happens between different diagrams and essentially relies on relations (2.1) and (2.2), while integral $B_{[-]}\left(q_{1}\right)=0$ due to the constraint (1.2). Therefore all power-like dependence on the rapidityregulator cancels in the leading power in $r$ and we are again left with single-logarithmic rapidity divergence related with gluon Reggeization.

\section{Conclusions and discussion}

In the present contribution we have reviewed the structure of rapidity divergences in the oneloop integrals contributing to the one-loop corrections to Particle-Particle-Reggeon effective vertices in the gauge-invariant EFT for Multi-Regge processes in QCD [1,2] and illustrated their application on two examples of such vertices: $\gamma^{\star} Q_{+} q$ and $g R_{+} g$. The first one contains two scales of virtuality: squared transverse momentum of Reggeized quark $t_{1}$ and virtuality of the photon $Q^{2}$, and new Lorentz structure $\Delta_{+\mu}^{(2)}$ appears in the answer for $Q^{2} \neq 0$. Cancellation of power-like dependence on rapidity regularization parameter $r$ is observed in both cases, so that only singlelogarithmic rapidity divergence is left in the end. 


\section{References}

[1] L. N. Lipatov, Nucl. Phys. B 452, 369, [hep-ph/9502308], (1995).

[2] L. N. Lipatov and M. I. Vyazovsky, Nucl. Phys. B 597, 399, [hep-ph/0009340], (2001).

[3] L. N. Lipatov, Phys. Rept. 286, 131, [hep-ph/9610276], (1997).

[4] S. Bondarenko and M. A. Zubkov, Eur. Phys. J. C 78, 617, [hep-ph/1801.08066], (2018).

[5] M. Hentschinski, Phys. Rev. D 97, 114027, [hep-ph/1802.06755], (2018).

[6] S. Bondarenko and S. Pozdnyakov, Int. J. Mod. Phys. A 33, 1850204, [hep-th/1806.02563], (2018) .

[7] S. Bondarenko, L. Lipatov, S. Pozdnyakov and A. Prygarin, Eur. Phys. J. C 77, 630, [hep-th/1708.05183], (2017).

[8] S. Bondarenko and S. Pozdnyakov, [hep-th/1903.11288], (2019).

[9] M. A. Nefedov, [hep-ph/1902.11030], (2019).

[10] M. Hentschinski, A. Sabio Vera, Phys. Rev. D85, 056006, [hep-ph/1110.6741] (2012); G. Chachamis, M. Hentschinski, J. D. Madrigal Martinez, A. Sabio Vera, Phys. Rev. D87, 076009, [hep-ph/1212.4992], (2013).

[11] M. Nefedov, V. Saleev, Mod. Phys. Lett. A32, 1750207, [hep-th/1709.06246], (2017).

[12] G. Chachamis, M. Hentschinski, J. D. Madrigal Martinez, A. Sabio Vera, Nucl. Phys. B876, 453-472, [hep-ph/1307.2591], (2013).

[13] R. K. Ellis, G. Zanderighi, JHEP 0802, 002, [hep-ph/0712.1851], (2008). 